\title{
Evaluation of Mucin-producing Gallbladder Carcinoma Using Magnetic Resonance Cholangiopancreatography: A Case Report
}

Logan Burstiner ${ }^{1}$, Bradley M. White ${ }^{2}$

1. Radiology, Larkin Community Hospital, Miami, USA 2. Interventional and Diagnostic Radiology, Larkin Community Hospital, Miami, USA

$\square$ Corresponding author: Logan Burstiner, lburstiner08054@med.lecom.edu Disclosures can be found in Additional Information at the end of the article

\section{Abstract}

Mucin-producing gallbladder carcinoma (MPGBC) is a rare and aggressive subtype of gallbladder carcinoma. We present a case of MPGBC with associated magnetic resonance imaging (MRI) / magnetic resonance cholangiopancreatography (MRCP) findings that may raise suspicion of this diagnosis preoperatively.

Categories: Pathology, Radiology, Gastroenterology

Keywords: mucin producing gallbladder carcinoma, mrcp

\section{Introduction}

In the United States, the incidence of gallbladder carcinoma is less than 5,000 cases per year [1]. Though this malignancy has several histological variants, mucin-producing gallbladder carcinoma (MPGBC) is a particularly rare and aggressive subtype. Recent studies suggest that it accounts for only $2.5 \%-5.5 \%$ of all gallbladder carcinomas [2-3].

MPGBC often presents with nonspecific, cholecystitis-like symptoms or is found incidentally during a cholecystectomy [4]. It can be difficult to differentiate from more benign entities using ultrasonography (US) or computed tomography (CT) alone [5-7]. As our case demonstrates, magnetic resonance imaging (MRI) / magnetic resonance cholangiopancreatography (MRCP) may be valuable in evaluating, and possibly diagnosing, MPGBC.

Received 04/04/2019 Review began 05/03/2019 Review ended 05/12/2019 Published 05/17/2019

\section{(C) Copyright 2019}

Burstiner et al. This is an open access article distributed under the terms of the Creative Commons Attribution License CCBY 3.0., which permits unrestricted use, distribution, and reproduction in any medium, provided the original author and source are credited.

\section{Case Presentation}

We present a 78-year-old male with a past medical history of dementia, coronary artery disease, hypertension, and chronic obstructive pulmonary disease (COPD) who presented to the emergency department for evaluation of severe jaundice and itching. He denied abdominal pain. His hypertransaminemia and hyperbilirubinemia suggested obstructive jaundice. Abdominal CT with oral contrast was nonspecific, showing gallbladder dilation and what appeared to be a simple calcified gallstone in the dependent portion of the fundus (Figures 1-2). Notably, what was likely the same stone was seen on a CT abdomen performed 10 years prior, but further imaging was not done at that time (Figure 3). 


\section{Cureus}

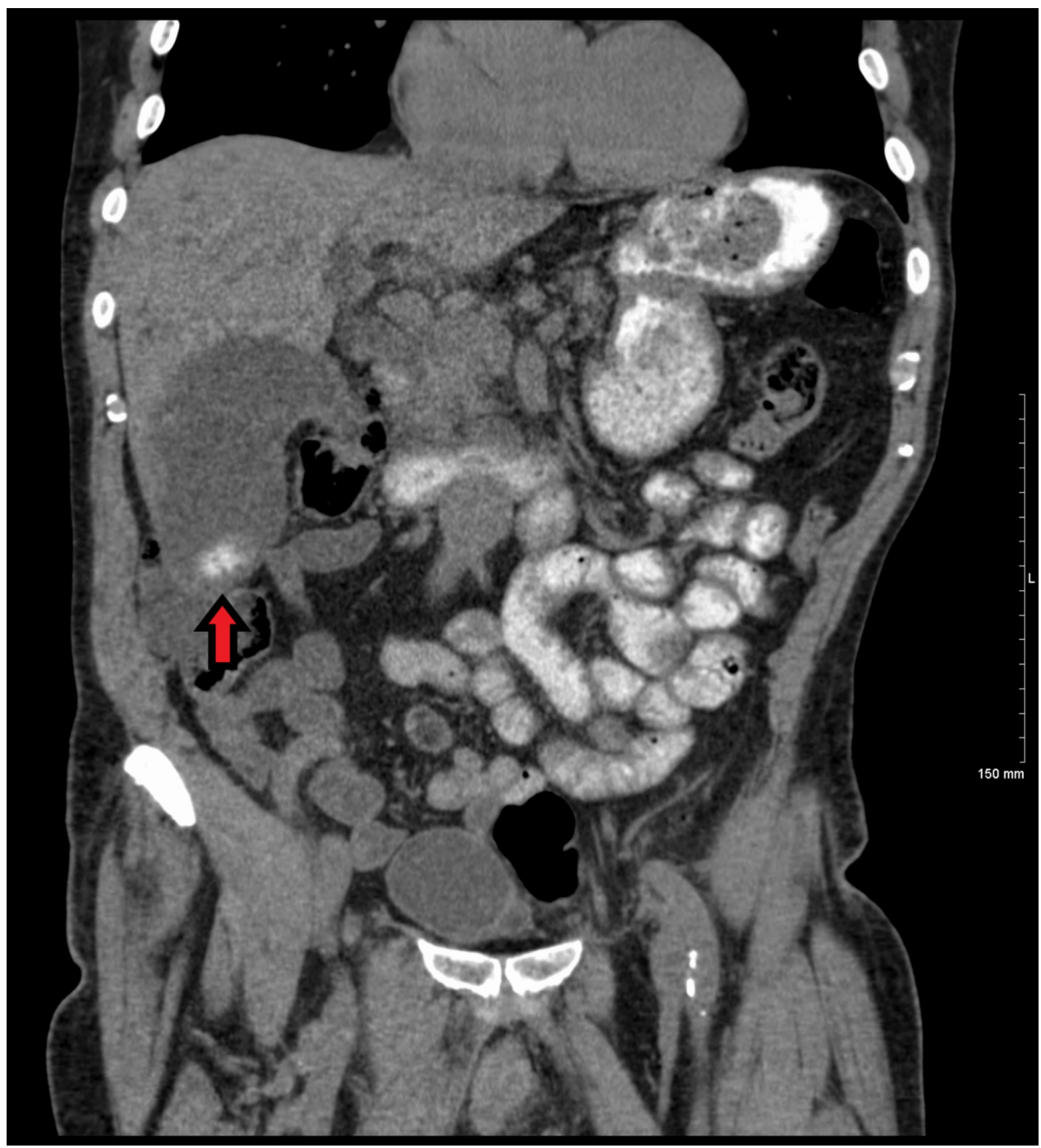

FIGURE 1: Coronal CT of the abdomen with oral contrast demonstrating a significantly dilated gallbladder. A $3 \mathrm{~cm}$ calcification is seen in the dependent portion of the fundus (arrow)

CT - Computed Tomography 


\section{Cureus}

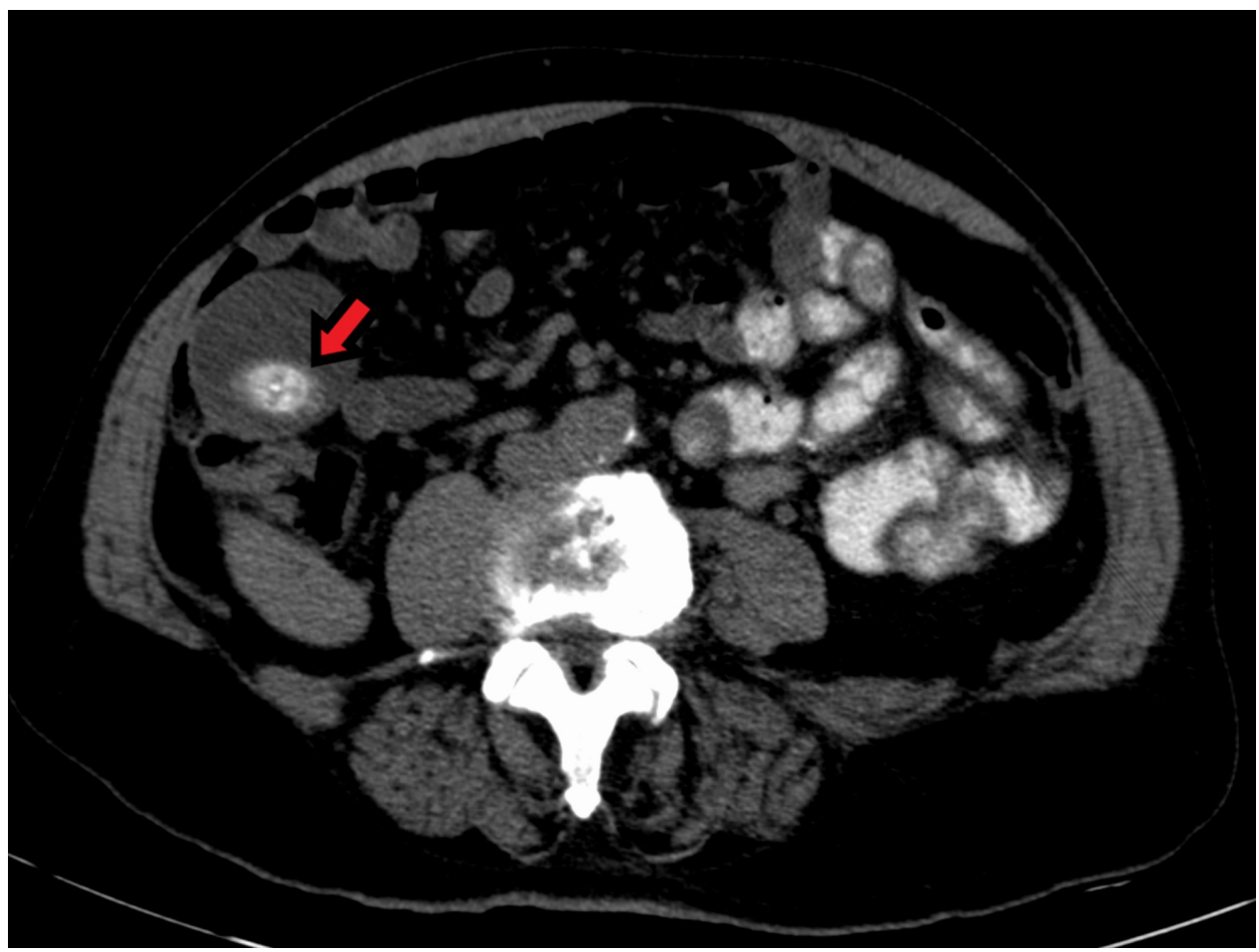

FIGURE 2: Axial view CT abdomen with oral contrast demonstrating a significantly dilated gallbladder. A $3 \mathrm{~cm}$ calcification is seen in the dependent portion of the fundus (arrow)

CT - Computed tomography

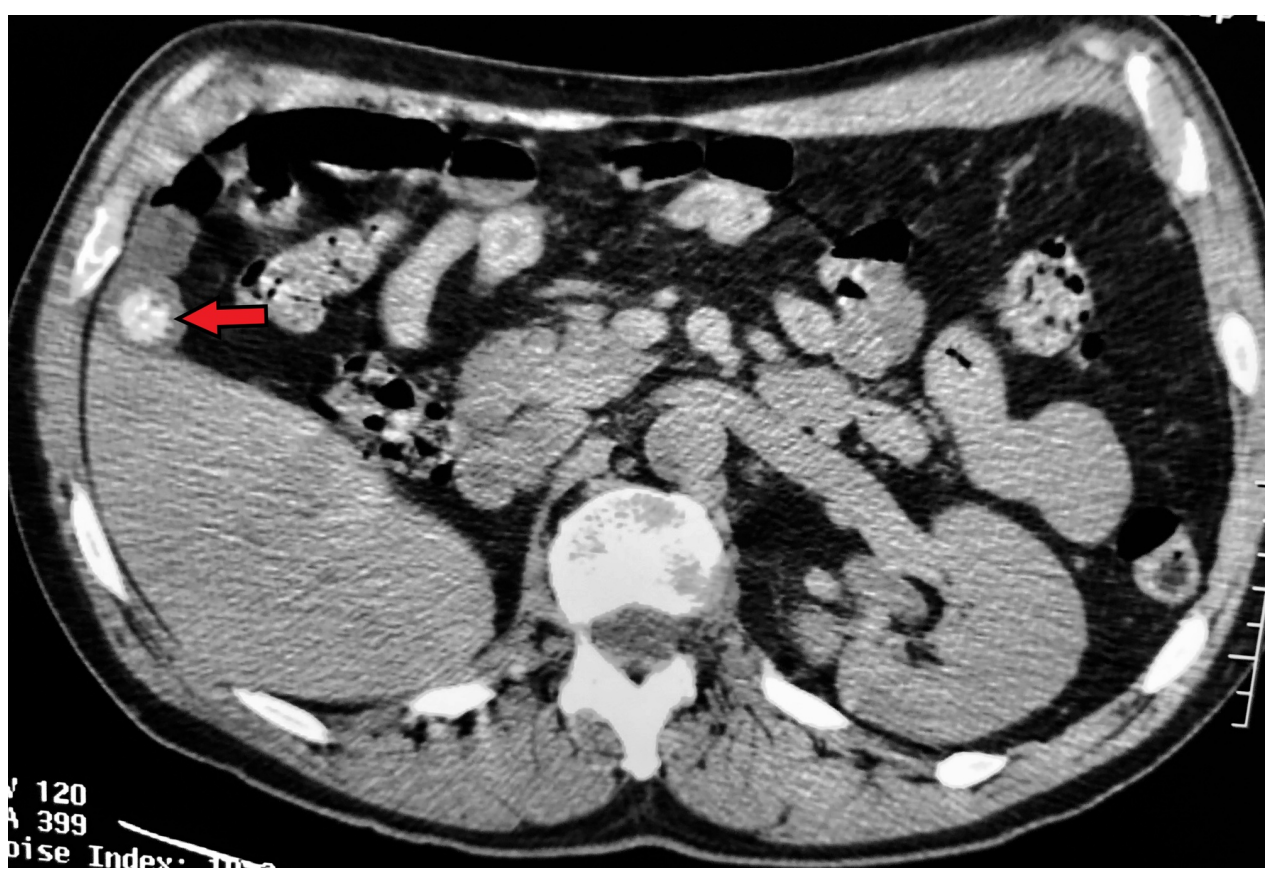

FIGURE 3: Axial CT of the abdomen without contrast taken 10 years prior. Prominent irregular calcification visible in the gallbladder fundus (arrow)

CT - Computed tomography 


\section{Cureus}

During this evaluation, follow-up US revealed irregular intraluminal masses that raised suspicions for malignancy (Figure 4). Subsequent MRI/MRCP clearly demonstrated a large, irregular mass surrounding the gallstone. Of note, hypointense curvilinear striations were visible along the long axis of the gallbladder (Figures 5-6). This characteristic finding has been seen before and was coined a "mucous thread" sign. It has been theorized to be the result of excessive viscous mucin produced by this carcinoma [8].

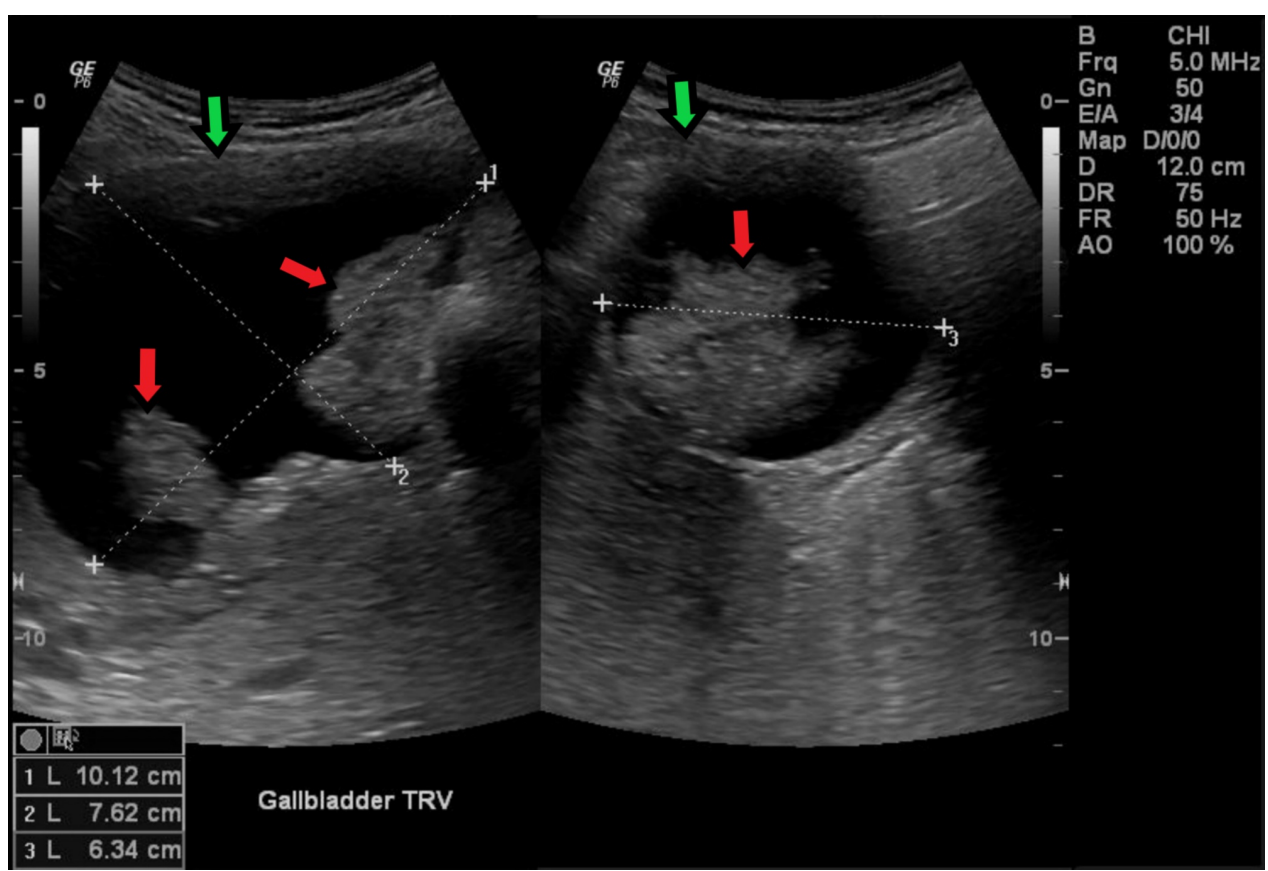

FIGURE 4: Transverse ultrasound of the gallbladder revealing multiple intraluminal masses (red arrows) and irregular wall thickening (green arrows). Gallbladder dimensions are given in the bottom left corner 


\section{Cureus}

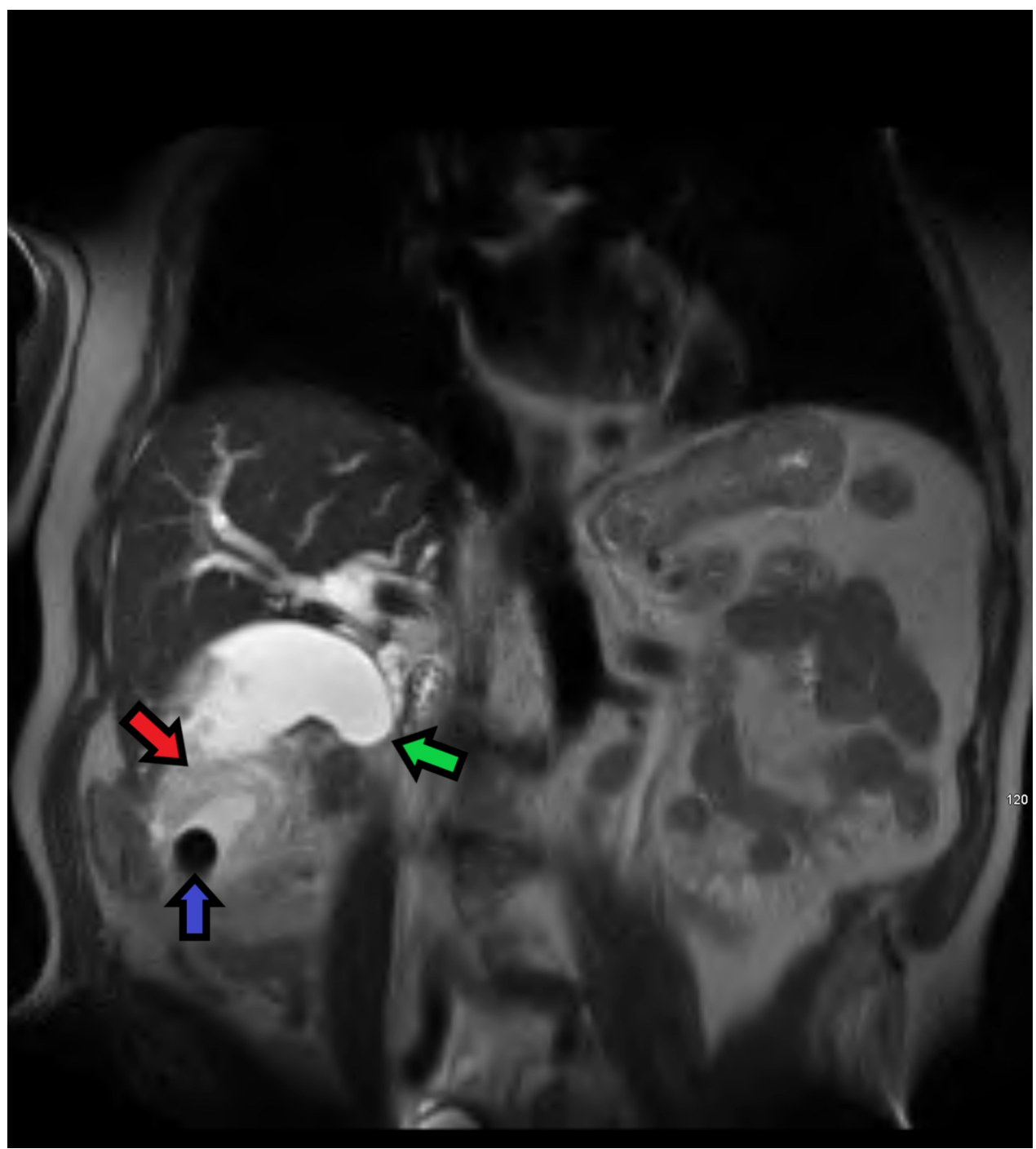

FIGURE 5: Coronal MRI T2 HASTE weighted image demonstrating a large mass (red arrow) surrounding a gallstone (blue arrow) in the distal portion of the gallbladder fundus. There is sharp tapering in the mid-todistal common bile duct (green arrow) indicating obstruction secondary to infiltration by the mass

MRI - Magnetic resonance image, HASTE - Half-Fourier Acquisition Single-shot Turbo Spin-Echo 


\section{Cureus}

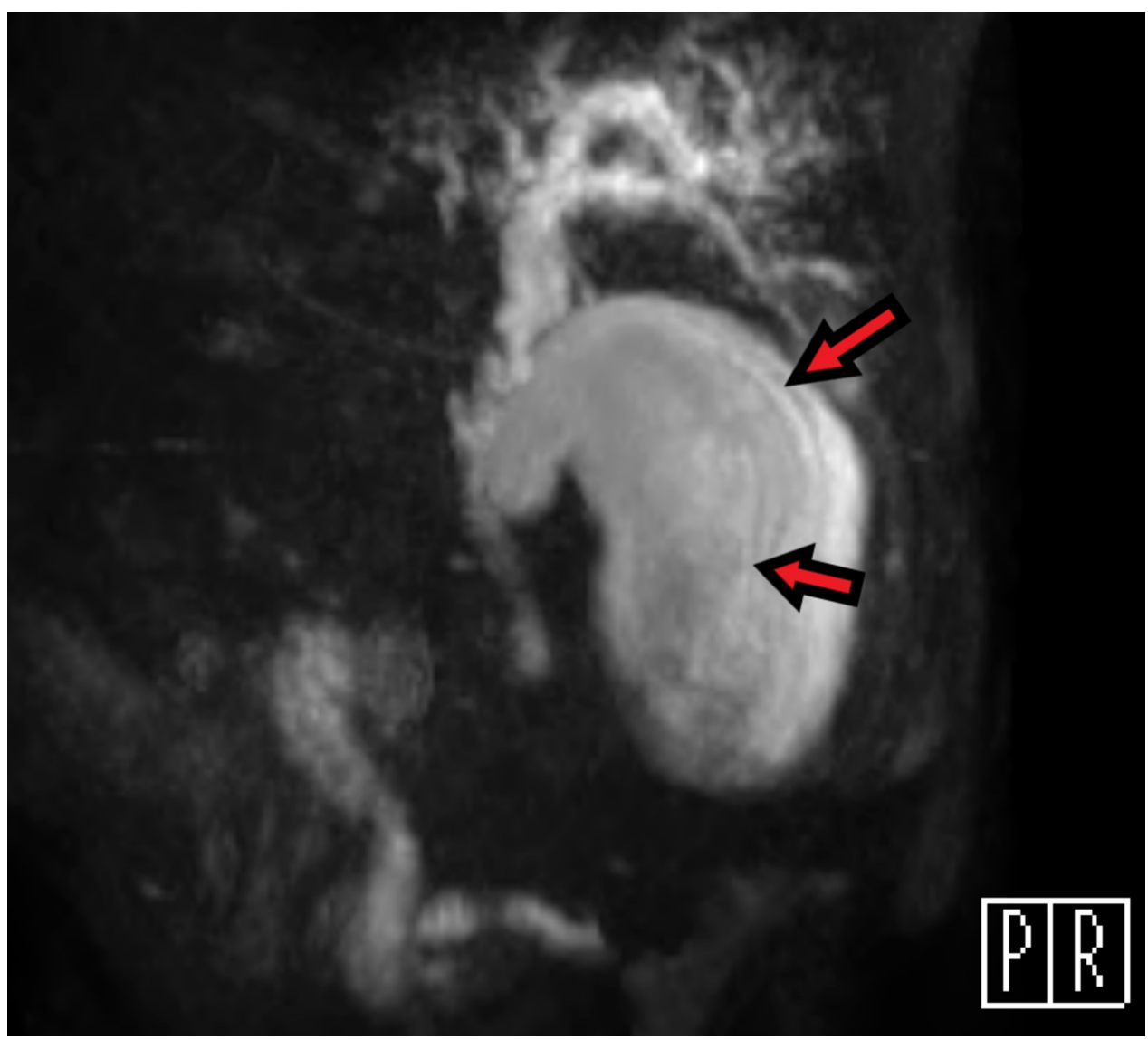

FIGURE 6: Oblique MRCP view of the gallbladder. Shows peripheral hypointense curvilinear striations parallel to the long axis (arrows) consistent with a mucous thread sign

MRCP - Magnetic resonance cholangiopancreatography

Though a laparoscopic cholecystectomy was performed, the area of invasion was too extensive for full resection. Histological samples confirmed MPGBC (Figure 7). The patient and his family declined further treatment of his malignancy, and he expired six months later. 


\section{Cureus}

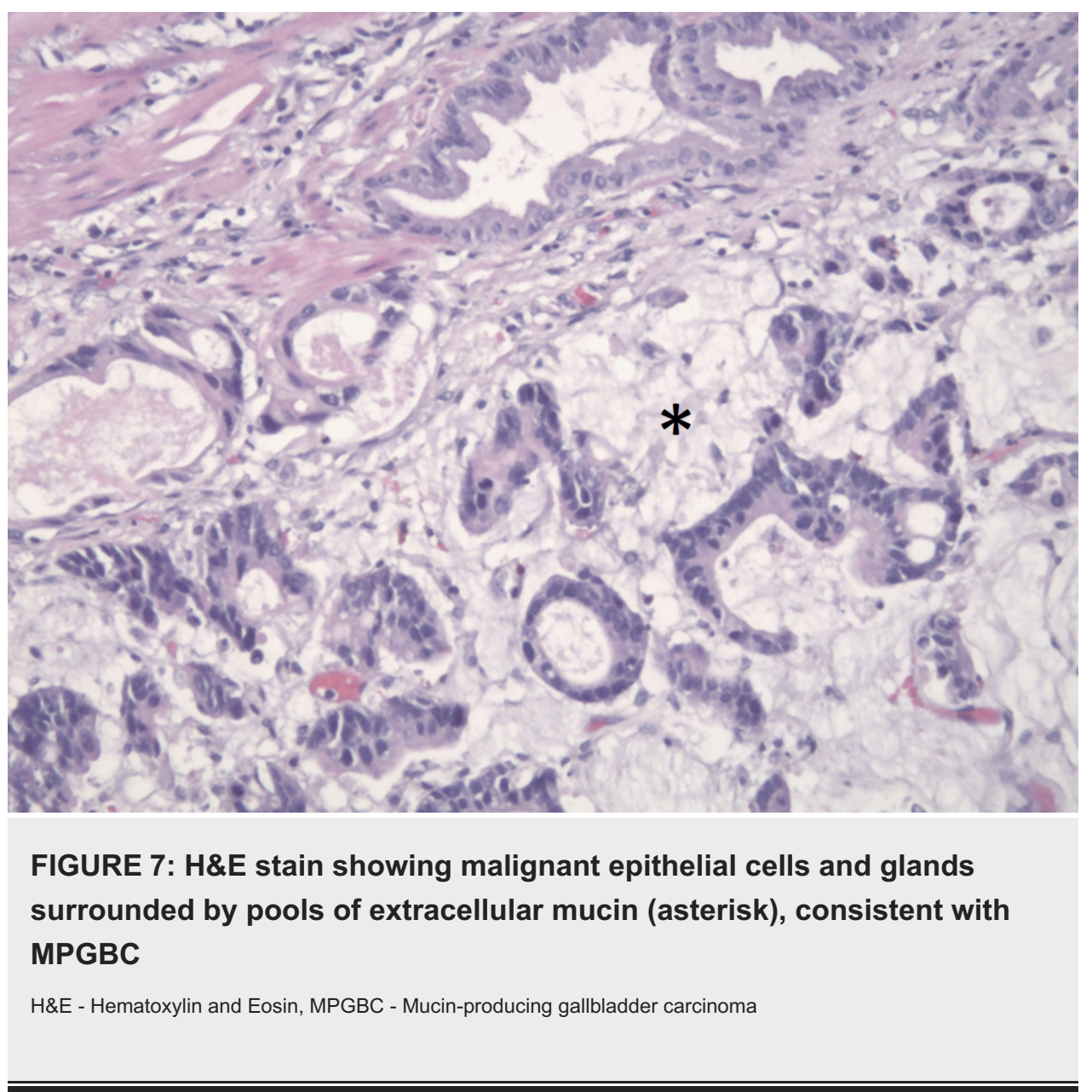

\section{Discussion}

MPGBC is extremely rare and can be overlooked or confused with less dangerous entities. It typically mimics acute cholecystitis and, therefore, most cases are diagnosed in the operating room during what is assumed to be a routine cholecystectomy. A careful imaging workup prior to surgery could inform clinicians and patients that they are dealing with a more malignant process. It is important for radiologists and other clinicians to be aware that the unique properties of this cancer may render some imaging modalities inferior to others.

Our case demonstrates the difficulty that CT can have in identifying MPGBC. Simply, a CT image is constructed based on the densities of different structures within the patient. Within the lumen of our patient's gallbladder, a hyperdense calcified gallstone is clearly visible. However, in these images, there is little evidence of a large mass surrounding the stone. Other researchers have described similar CT findings in cases of MPGBC. They have speculated that the excessive amount of mucin produced within the tumor may give it a density near to that of water; obscuring the mass by making it impossible to differentiate from fluid already within the gallbladder [7].

Ultrasonography is the initial imaging test of choice for any patient with possible gallbladder disease. Benefits include low cost and ease of use. In most cases, it adequately identifies masses in the gallbladder. However, it is less reliable in determining whether the mass is benign or malignant. It is inferior to other imaging modalities (CT and MRI) for identifying and staging gallbladder cancer. Due to this, current guidelines recommend following up any suspicious US findings with either a CT or an MRI [5-7].

As previously discussed, the MRI for our patient was much more informative than the other imaging techniques. While the CT could only visualize a gallstone, the MRI clearly depicted the large mass totally enveloping the stone and filling the gallbladder. At this point in the evaluation, a malignant process was obviously strongly suspected. Likely due to the rarity of MPGBC, it still came as a surprise when the intraoperative pathology report returned with this diagnosis.

Our retrospective investigation revealed that the MRCP may have suggested the lesion was this specific subtype of gallbladder cancer. It is possible that the hypointense striations along the long axis of the patient's gallbladder are unique radiographic signs of MPGBC. To our knowledge, there is no other case 


\section{Cureus}

study discussing MRCP evaluation of MPGBC in United States literature. Japanese researchers, however, identified multiple patients with MPGBC who had similar linear hypointense areas visible on MRCP. They considered that the highly viscous properties of the mucin were responsible for the appearance of these characteristic striations and named this a "mucous thread" sign [8].

\section{Conclusions}

Our case highlights the limitations of CT and US, as well as the value of MRI/MRCP, in the diagnostic evaluation of MPGBC. In addition, we draw attention to a unique radiographic finding of MPGBC (mucous thread sign) that may lead to the early detection of this highly fatal disease.

\section{Additional Information}

\section{Disclosures}

Human subjects: Consent was obtained by all participants in this study. Conflicts of interest: In compliance with the ICMJE uniform disclosure form, all authors declare the following: Payment/services info: All authors have declared that no financial support was received from any organization for the submitted work. Financial relationships: All authors have declared that they have no financial relationships at present or within the previous three years with any organizations that might have an interest in the submitted work. Other relationships: All authors have declared that there are no other relationships or activities that could appear to have influenced the submitted work.

\section{Acknowledgements}

Dr. Maryam Sharifian, David Costello

\section{References}

1. Carriaga MT, Henson DE: Liver, gallbladder, extrahepatic bile ducts, and pancreas. Cancer. 1995, 75:171190.

2. Dursun N, Escalona OT, Roa JC, et al.: Mucinous carcinoma of the gallbladder: clinicopathologic analysis of 15 cases identified in 606 carcinomas. Arch Pathol Lab Med. 2012, 136:1347-1358. 10.5858/arpa.2011-0447$\mathrm{OA}$

3. Yadav R, Jain D, Mathur SR, Sharma A, Iyer VK: Gallbladder carcinoma: an attempt of WHO histological classification on fine needle aspiration material. Cytojournal. 2013, 10:12. 10.4103/1742-6413.113627

4. Levy AD, Murakata LA, Rohrmann CA Jr: Gallbladder carcinoma: radiologic-pathologic correlation. RadioGraphics. 2001, 21:295-314. 10.1148/radiographics.21.2.g01mr16295

5. Valle JW, Borbath I, Khan SA, Huguet F, Gruenberger T, Arnold D: Biliary cancer: ESMO Clinical Practice Guidelines for diagnosis, treatment and follow-up. Ann Oncol. 2016, 27:28-37. 10.1093/annonc/mdw324

6. National Comprehensive Cancer Network. Gallbladder cancer. (2018). Accessed: May 17, 2019: https://www.nccn.org/patients/guidelines/hepatobiliary/files/assets/common/downloads/SurveyQuickGuide waldens

7. Huang CP, Chiou YY, Chou YH, Chiang JH, Chang CY: Imaging findings in mucin-producing carcinoma of the gallbladder. J Formos Med Assoc. 2006, 105:427-430. 10.1016/S0929-6646(09)60141-8

8. Ishiguro S, Onaya H, Esaki M, et al.: Mucin-producing carcinoma of the gallbladder: evaluation by magnetic resonance cholangiopancreatography in three cases. Korean J Radiol. 2012, 13:637-642. 10.3348/kjr.2012.13.5.637 